\title{
BOUNDS AND ASYMPTOTIC EXPANSIONS FOR THE DISTRIBUTION OF THE MAXIMUM OF A SMOOTH STATIONARY GAUSSIAN PROCESS *
}

\author{
Jean-Marc Azaïs ${ }^{1}$, Christine Cierco-Ayrolles ${ }^{1,2}$ And Alain Croquette ${ }^{1}$
}

\begin{abstract}
This paper uses the Rice method [18] to give bounds to the distribution of the maximum of a smooth stationary Gaussian process. We give simpler expressions of the first two terms of the Rice series $[3,13]$ for the distribution of the maximum. Our main contribution is a simpler form of the second factorial moment of the number of upcrossings which is in some sense a generalization of Steinberg et al.'s formula ([7] p. 212). Then, we present a numerical application and asymptotic expansions that give a new interpretation of a result by Piterbarg [15].

Résumé. Dans cet article nous utilisons la méthode de Rice (Rice, 1944-1945) pour trouver un encadrement de la fonction de répartition du maximum d'un processus Gaussien stationnaire régulier. Nous dérivons des expressions simplifiées des deux premiers termes de la série de Rice (Miroshin, 1974, Azaïs et Wschebor, 1997) suffisants pour l'encadrement cherché. Notre contribution principale est la donnée d'une forme plus simple du second moment factoriel du nombre de franchissements vers le haut, ce qui est, en quelque sorte, une généralisation de la formule de Steinberg et al. (Cramér and Leadbetter, 1967, p. 212). Nous présentons ensuite une application numérique et des développements asymptotiques qui fournissent une nouvelle interprétation d'un résultat de Piterbarg (1981).
\end{abstract}

AMS Subject Classification. 60Exx, 60Gxx, 60G10, 60G15, 60G70, 62E17, 65U05.

Received June 4, 1998. Revised June 8, 1999.

\section{INTRODUCTION}

\subsection{Framework}

Many statistical models involve nuisance parameters. This is the case for example for mixture models [10], gene detection models [5,6], projection pursuit [20]. In such models, the distributions of test statistics are those of the maximum of stochastic Gaussian processes (or their squares). Dacunha-Castelle and Gassiat [8] give for example a theory for the so-called "locally conic models".

Thus, the calculation of threshold or power of such tests leads to the calculation of the distribution of the maximum of Gaussian processes. This problem is largely unsolved [2].

Keywords and phrases: Asymptotic expansions, extreme values, stationary Gaussian process, Rice series, upcrossings.

* This paper is dedicated to Mario Wschebor in the occasion of his 60th birthday.

${ }^{1}$ Laboratoire de Statistique et Probabilités, UMR C55830 du CNRS, Université Paul Sabatier, 118 route de Narbonne, 31062

Toulouse Cedex 4, France.

2 Institut National de la Recherche Agronomique, Unité de Biométrie et Intelligence Artificielle, BP. 27, Chemin de Borde-Rouge, 31326 Castanet-Tolosan Cedex, France; e-mail: azais@cict.fr, cierco@toulouse.inra.fr, croquett@cict.fr 
Miroshin [13] expressed the distribution function of this maximum as a sum of a series, so-called the "Rice series". Recently, Azaïs and Wschebor [3,4] proved the convergence of this series under certain conditions and proposed a method giving the exact distribution of the maximum for a class of processes including smooth stationary Gausian processes with real parameter.

The formula given by the Rice series is rather complicated, involving multiple integrals with complex expressions. Fortunatly, for some processes, the convergence is very fast, so the present paper studies the bounds given by the first two terms that are in some cases sufficient for application.

We give identities that yield simpler expressions of these terms in the case of stationary processes. Generalization to other processes is possible using our techniques but will not be detailed for shortness and simplicity.

For other processes, the calculation of more than two terms of the Rice series is necessary. In such a case, the identities contained in this paper (and other similar) give a list of numerical tricks used by a program under construction by Croquette.

We then use Maple to derive asymptotic expansions of some terms involved in these bounds. Our bounds are shown to be sharp and our expansions are made for a fixed time interval and a level tending to infinity. Other approaches can be found in the literature [12]. For example, Kratz and Rootzén [11] propose asymptotic expansions for a size of time interval and a level tending jointly to infinity.

We consider a real valued centred stationary Gaussian process with continuous paths $X=\left\{X_{t} ; t \in[0, T] \subset \mathbb{R}\right\}$. We are interested in the random variables

$$
X^{\star}=\sup _{t \in[0, T]} X_{t} \text { or } X^{\star \star}=\sup _{t \in[0, T]}\left|X_{t}\right| .
$$

For shortness and simplicity, we will focus attention on the variable $X^{\star}$; the necessary modifications for adapting our method to $X^{\star \star}$ are easy to establish [5].

We denote by $d F(\lambda)$ the spectral measure of the process $X$ and $\lambda_{p}$ the spectral moment of order $p$ when it exists. The spectral measure is supposed to have a finite second moment and a continuous component. This implies ([7] p. 203) that the process is differentiable in quadratic mean and that for all pairwise different time points $t_{1}, \ldots, t_{n}$ in $[0, T]$, the joint distribution of $X_{t_{1}}, \ldots, X_{t_{n}}, X_{t_{1}}^{\prime}, \ldots, X_{t_{n}}^{\prime}$ is non degenerated.

For simplicity, we will assume that moreover the process admits $\mathcal{C}^{1}$ sample paths. We will denote by $r($.$) the$ covariance function of $X$ and, without loss of generality, we will suppose that $\lambda_{0}=r(0)=1$.

Let $u$ be a real number, the number of upcrossings of the level $u$ by $X$, denoted by $U_{u}$ is defined as follows:

$$
U_{u}=\#\left\{t \in[0, T], X_{t}=u, X_{t}^{\prime}>0\right\} .
$$

For $k \in \mathbb{N}^{\star}$, we denote by $\nu_{k}(u, T)$ the factorial moment of order $k$ of $U_{u}$ and by $\tilde{\nu}_{k}(u, T)$ the factorial moment of order $k$ of $U_{u} \mathbb{1}_{\left\{X_{0} \leq u\right\}}$. We also define $\bar{\nu}_{k}(u, T)=\nu_{k}(u, T)-\tilde{\nu}_{k}(u, T)$. These factorial moments can be calculated by Rice formulae. For example:

$$
\begin{gathered}
\nu_{1}(u, T)=\mathbb{E}\left(U_{u}\right)=\frac{T \sqrt{\lambda_{2}}}{2 \pi} e^{-u^{2} / 2} \\
\text { and } \nu_{2}(u, T)=\mathbb{E}\left(U_{u}\left(U_{u}-1\right)\right)=\int_{0}^{T} \int_{0}^{T} A_{s-t}(u) d s d t
\end{gathered}
$$

with $A_{s-t}(u)=\mathbb{E}\left(\left(X_{s}^{\prime}\right)^{+}\left(X_{t}^{\prime}\right)^{+} \mid X_{s}=X_{t}=u\right) p_{s, t}(u, u)$, where $\left(X_{\bullet}^{\prime}\right)^{+}$is the positive part of $X_{\bullet}^{\prime}$ and $p_{s, t}$ the joint density of $\left(X_{s}, X_{t}\right)$.

These two formulae are proved to hold under our hypotheses ([7], p. 204). See also Wschebor [21], Chapter 3, for the case of more general processes.

We will denote by $\varphi$ the density of the standard Gaussian distribution. In order to have simpler expressions of rather complicated formulae, we will use the folllowing three functions: $\Phi(x)=\int_{-\infty}^{x} \varphi(y) d y, \bar{\Phi}(x)=1-\Phi(x)$ and $\Psi(x)=\int_{0}^{x} \varphi(y) d y=\Phi(x)-\frac{1}{2}$. 


\subsection{Main inequalities}

Since the pioneering works of Rice [18], the most commonly used upper bound for the distribution of the maximum is the following:

$$
P\left(X^{\star}>u\right) \leq P\left(X_{0}>u\right)+P\left(U_{u}>0\right) \leq P\left(X_{0}>u\right)+\mathbb{E}\left(U_{u}\right) .
$$

That is: $P\left(X^{\star}>u\right) \leq \bar{\Phi}(u)+T \sqrt{\frac{\lambda_{2}}{2 \pi}} \varphi(u)$.

One can also see the works by $[9,15,16]$.

We propose here a slight refinement of this inequality, but also a lower bound using the second factorial moment of the number of upcrossings. Our results are based on the following remark which is easy to check: if $\xi$ is a non-negative integer valued random variable, then

$$
\mathbb{E}(\xi)-\frac{1}{2} \mathbb{E}(\xi(\xi-1)) \leq P(\xi>0) \leq \mathbb{E}(\xi) .
$$

Noting that $P$ almost surely, $\left\{X^{\star}>u\right\}=\left\{X_{0}>u\right\} \cup\left\{X_{0} \leq u, U_{u}>0\right\}$ and that $\mathbb{E}\left(U_{u}\left(U_{u}-1\right) \mathbb{1}_{\left\{X_{0} \leq u\right\}}\right) \leq \nu_{2}$, we get:

$$
P\left(X_{0}>u\right)+\tilde{\nu}_{1}(u, T)-\frac{\nu_{2}(u, T)}{2} \leq P\left(X^{\star} \geq u\right) \leq P\left(X_{0}>u\right)+\tilde{\nu}_{1}(u, T),
$$

with $\tilde{\nu}_{1}(u, T)=\mathbb{E}\left\{U_{u} \mathbb{1}_{\left\{X_{0} \leq u\right\}}\right\}$.

Using the same technique as for calculating $\mathbb{E}\left(U_{u}\right)$ and $\mathbb{E}\left(U_{u}\left(U_{u}-1\right)\right)$, one gets

$$
\tilde{\nu}_{1}(u, T)=\int_{0}^{T} d t \int_{-\infty}^{u} d x \int_{0}^{+\infty} y p_{0, t ; t}(x, u ; y) d y
$$

where $p_{0, t ; t}$ stands for the density of the vector $\left(X_{0}, X_{t}, X_{t}^{\prime}\right)$.

Azaïs and Wschebor [3,4] have proved, under certain conditions, the convergence of the Rice series [13]

$$
P\left(X^{\star} \geq u\right)=P\left(X_{0}>u\right)+\sum_{m=1}^{+\infty}(-1)^{m+1} \frac{\tilde{\nu}_{m}(u, T)}{m !}
$$

and the envelopping property of this series:

if we set $S_{n}=P\left(X_{0}>u\right)+\sum_{m=1}^{n}(-1)^{m+1} \frac{\tilde{\nu}_{m}(u, T)}{m !}$, then, for all $n>0$ :

$$
S_{2 n} \leq P\left(X^{\star} \geq u\right) \leq S_{2 n-1}
$$

Using relation (1.3) with $n=1$ gives

$$
P\left(X_{0}>u\right)+\tilde{\nu}_{1}(u, T)-\frac{\tilde{\nu}_{2}(u, T)}{2} \leq P\left(X^{\star} \geq u\right) \leq P\left(X_{0}>u\right)+\tilde{\nu}_{1}(u, T) .
$$

Since $\tilde{\nu}_{2}(u, T) \leq \nu_{2}(u, T)$, we see that, except this last modification which gives a simpler expression, Main inequality (1.1) is relation (1.3) with $n=1$. 
Remark 1.1. In order to calculate these bounds, we are interested in the quantity $\tilde{\nu}_{1}(u, T)$. For asymptotic calculations and to compare our results with Piterbarg's ones, we will also consider the quantity $\bar{\nu}_{k}(u, T)$. From a numerical point of view, $\bar{\nu}_{k}(u, T)$ and $\tilde{\nu}_{k}(u, T)$ are worth being distinguished because they are not of same order of magnitude as $u \rightarrow+\infty$. In the following sections, we will work with $\bar{\nu}_{1}(u, T)$.

\section{Some identities}

First, let us introduce some notations that will be used in the rest of the paper. We set:

- $\mu(t)=\mathbb{E}\left(X_{0}^{\prime} \mid X_{0}=X_{t}=u\right)=-\frac{r^{\prime}(t)}{1+r(t)} u$,

- $\sigma^{2}(t)=\operatorname{Var}\left(X_{0}^{\prime} \mid X_{0}=X_{t}=u\right)=\lambda_{2}-\frac{r^{\prime 2}(t)}{1-r^{2}(t)}$,

- $\rho(t)=\operatorname{Cor}\left(X_{0}^{\prime}, X_{t}^{\prime} \mid X_{0}=X_{t}=u\right)=\frac{-r^{\prime \prime}(t)\left(1-r^{2}(t)\right)-r(t) r^{\prime 2}(t)}{\lambda_{2}\left(1-r^{2}(t)\right)-r^{\prime 2}(t)}$.

We also define $k(t)=\sqrt{\frac{1+\rho(t)}{1-\rho(t)}}$ and $b(t)=\frac{\mu}{\sigma}(t)$.

Note that, since the spectrum of the process $X$ admits a continuous component, $|\rho(t)| \neq 1$.

In the sequel, the variable $t$ will be omitted when it is not confusing and we will write $r, r^{\prime}, \mu, \sigma, \rho, k, b$ instead of $r(t), r^{\prime}(t), \mu(t), \sigma(t), \rho(t), k(t), b(t)$.

Proposition 2.1. (i) If $(X, Y)$ has a centred normal bivariate distribution with covariance matrix $\left(\begin{array}{cc}1 & \rho \\ \rho & 1\end{array}\right)$, then $\forall a \in \mathbb{R}^{+}$

$$
\begin{aligned}
P(X>a, Y>-a) & =\frac{1}{\pi} \arctan \left(\sqrt{\frac{1+\rho}{1-\rho}}\right)-2 \int_{0}^{a} \varphi(x) \Psi\left(\sqrt{\frac{1+\rho}{1-\rho}} x\right) d x \\
& =2 \int_{a}^{+\infty} \Psi\left(\sqrt{\frac{1+\rho}{1-\rho}} x\right) \varphi(x) d x
\end{aligned}
$$

(ii) $\overline{\nu_{1}}(u, T)=\varphi(u) \int_{0}^{T}\left(\sqrt{\frac{\lambda_{2}}{2 \pi}} \Phi\left(\sqrt{\frac{1-r}{1+r}} \frac{\sqrt{\lambda_{2}}}{\sigma} u\right)+\varphi\left(\sqrt{\frac{1-r}{1+r}} u\right) \bar{\Phi}(b) \frac{r^{\prime}}{\sqrt{1-r^{2}}}\right) d t$

(iii) $\nu_{2}(u, T)=\int_{0}^{T} 2(T-t) \frac{1}{\sqrt{1-r^{2}(t)}} \varphi^{2}\left(\frac{u}{\sqrt{1+r(t)}}\right)\left[T_{1}(t)+T_{2}(t)+T_{3}(t)\right] d t$

with:

$$
\begin{gathered}
T_{1}(t)=\sigma^{2}(t) \sqrt{1-\rho^{2}(t)} \varphi(b(t)) \varphi(k(t) b(t)), \\
T_{2}(t)=2\left(\sigma^{2}(t) \rho(t)-\mu^{2}(t)\right) \int_{b(t)}^{+\infty} \Psi(k(t) x) \varphi(x) d x, \\
T_{3}(t)=2 \mu(t) \sigma(t) \Psi(k(t) b(t)) \varphi(b(t)) .
\end{gathered}
$$

(iv) A second expression for $T_{2}(t)$ is:

$$
T_{2}(t)=\left(\sigma^{2}(t) \rho(t)-\mu^{2}(t)\right)\left[\frac{1}{\pi} \arctan (k(t))-2 \int_{0}^{b(t)} \Psi(k(t) x) \varphi(x) d x\right] .
$$


Remark 2.2. 1. Formula ( $i)$ is analogous to the formula (2.10.4) given in Cramér and Leadbetter's [7], p. 27:

$$
P(X>a, Y>-a)=\Phi(a) \bar{\Phi}(a)+\int_{0}^{\rho} \frac{1}{2 \pi \sqrt{1-z^{2}}} \exp \left(-\frac{a^{2}}{1-z}\right) d z .
$$

Our formula is easier to prove and is more adapted to numerical application because, when $t \rightarrow 0$, $\rho(t) \rightarrow-1$ and the integrand in Cramér and Leadbetter's formula tends to infinity.

2. Utility of these formulae:

- these formulae permit a computation of Main inequality (1.1), at the cost of a double integral with finite bounds. This is a notable reduction of complexity with respect to the original form. The form (2.4) is more adapted to effective computation, because it involves an integral on a bounded interval;

- this method has been implemented in a $\mathrm{S}+$ program that needs about one second of Cpu to run an example. It has been applied to a genetical problem in Cierco and Azaïs [6].

The form (iii) has some consequences both for numerical and theoretical purposes. The calculation of $\nu_{2}(u, T)$ yields some numerical difficulties around $t=0$. The sum of the three terms is infinitly small with respect to each term. To discard the diagonal from the computation, we use formula (iii) and Maple to calculate the equivalent of the integrand in the neighbourhood of $t=0$ at fixed $u$.

Recall that we have set $\nu_{2}(u, T)=\int_{0}^{T} \int_{0}^{T} A_{s-t}(u) d s d t$. The following proposition gives the Taylor expansion of $A$ at zero.

Proposition 2.3. Assume that $\lambda_{8}$ is finite. Then, as $t \rightarrow 0$ :

$$
A_{t}(u)=\frac{1}{1296} \frac{\left(\lambda_{2} \lambda_{6}-\lambda_{4}\right)^{3 / 2}}{\left(\lambda_{4}-\lambda_{2}^{2}\right)^{1 / 2} \pi^{2} \lambda_{2}^{2}} \exp \left(-\frac{1}{2} \frac{\lambda_{4}}{\lambda_{4}-\lambda_{2}^{2}} u^{2}\right) t^{4}+O\left(t^{5}\right) .
$$

Piterbarg [17] or Wschebor [21] proved that $A_{t}(u)=O(\varphi(u(1+\delta)))$ for some $\delta \rightarrow 0$. Our result is more precise.

Our formulae give some asymptotic expansions as $u \rightarrow+\infty$ for $\bar{\nu}_{1}(u, T)$ and $\nu_{2}(u, T)$ for small $T$.

Proposition 2.4. Assume that $\lambda_{8}$ is finite. Then, there exists a value $T_{0}$ such that, for every $T<T_{0}$

$$
\begin{aligned}
& \bar{\nu}_{1}(u, T)=\frac{27}{4 \sqrt{\pi}} \frac{\left(\lambda_{4}-\lambda_{2}^{2}\right)^{11 / 2}}{\lambda_{2}^{5}\left(\lambda_{2} \lambda_{6}-\lambda_{4}^{2}\right)^{3 / 2}} \varphi\left(\sqrt{\frac{\lambda_{4}}{\lambda_{4}-\lambda_{2}^{2}}} u\right) u^{-6}\left(1+O\left(\frac{1}{u}\right)\right) \\
& \nu_{2}(u, T)=\frac{3 \sqrt{3} T}{\pi} \frac{\left(\lambda_{4}-\lambda_{2}^{2}\right)^{9 / 2}}{\lambda_{2}^{9 / 2}\left(\lambda_{2} \lambda_{6}-\lambda_{4}^{2}\right)} \varphi\left(\sqrt{\frac{\lambda_{4}}{\lambda_{4}-\lambda_{2}^{2}}} u\right) u^{-5}\left(1+O\left(\frac{1}{u}\right)\right)
\end{aligned}
$$

as $u \longrightarrow+\infty$.

\section{A NUMERICAL EXAMPLE}

In the following example, we show how the upper and lower bounds (1.1) permit to evaluate the distribution of $X^{\star}$ with an error less than $10^{-4}$.

We consider the centered stationary Gaussian process with covariance $\Gamma(t):=\exp \left(-t^{2} / 2\right)$ on the interval $I=[0,1]$, and the levels $u=-3,-2.5, \ldots, 3$. The term $P\left(X_{0} \leq u\right)$ is evaluated by the $S$-plus function Pnorm, $\tilde{\nu_{1}}$ and $\nu_{2}$ using Proposition 2.1 and the Simpson method. Though it is rather difficult to assess the exact precision of these evaluations, it is clear that it is considerably smaller than $10^{-4}$. So, the main source of error 
is due to the difference between the upper and lower bounds in (1.1).

$\begin{array}{lccccc}u & P\left(X_{0} \leq u\right) & \tilde{\nu}_{1} & \nu_{2} & \text { lower bound } & \text { upper bound } \\ -3 & 0.00135 & 0.00121 & 0 & 0.00014 & 0.00014 \\ -2.5 & 0.00621 & 0.00518 & 0 & 0.00103 & 0.00103 \\ -2 & 0.02275 & 0.01719 & 0 & 0.00556 & 0.00556 \\ -1.5 & 0.06681 & 0.04396 & 0.00001 & 0.02285 & 0.02285 \\ -1 & 0.15866 & 0.08652 & 0.00002 & 0.07213 & 0.07214 \\ -0.5 & 0.30854 & 0.13101 & 0.00004 & 0.17753 & 0.17755 \\ 0 & 0.50000 & 0.15272 & 0.00005 & 0.34728 & 0.34731 \\ 0.5 & 0.69146 & 0.13731 & 0.00004 & 0.55415 & 0.55417 \\ 1 & 0.84134 & 0.09544 & 0.00002 & 0.74591 & 0.74592 \\ 1.5 & 0.93319 & 0.05140 & 0.00001 & 0.88179 & 0.88180 \\ 2 & 0.97725 & 0.02149 & 0 & 0.95576 & 0.95576 \\ 2.5 & 0.99379 & 0.00699 & 0 & 0.98680 & 0.98680 \\ 3 & 0.99865 & 0.00177 & 0 & 0.99688 & 0.99688\end{array}$

The calculation demands $14 \mathrm{~s}$ on a Pentium $100 \mathrm{MHz}$.

The corresponding program is available sending an e-mail to croquett@cict.fr.

\section{Proofs}

Proof of Proposition 2.1

Proof of point (i). We first search $P(X>a, Y>a)$.

Put $\rho=\cos (\theta), \theta \in\left[0, \pi\left[\right.\right.$, and use the orthogonal decomposition $Y=\rho X+\sqrt{1-\rho^{2}} Z$.

Then $\{Y>a\}=\left\{Z>\frac{a-\rho X}{\sqrt{1-\rho^{2}}}\right\}$. Thus:

$$
P(X>a, Y>a)=\int_{a}^{+\infty} \varphi(x) \bar{\Phi}\left(\frac{a-\rho x}{\sqrt{1-\rho^{2}}}\right) d x=\iint_{\mathcal{D}} \varphi(x) \varphi(z) d x d z
$$

where $\mathcal{D}$ is the domain located between the two half straight lines starting from the point $\left(a, a \sqrt{\frac{1-\rho}{1+\rho}}\right)$ and with angle $\theta-\frac{\pi}{2}$ and $\frac{\pi}{2}$.

Using a symmetry with respect to the straight line with angle $\frac{\theta}{2}$ passing through the origin, we get:

$$
P(X>a, Y>a)=2 \int_{a}^{+\infty} \varphi(x) \bar{\Phi}\left(\sqrt{\frac{1-\rho}{1+\rho}} x\right) d x .
$$

Now,

$$
P(X>a, Y>-a)=\bar{\Phi}(a)-P(X>a, Y<-a)=\bar{\Phi}(a)-P(X>a,(-Y)>a) .
$$

Applying relation (4.1) to $(X,-Y)$ yields

$$
P(X>a, Y>-a)=\bar{\Phi}(a)-2 \int_{a}^{+\infty} \varphi(x) \bar{\Phi}\left(\sqrt{\frac{1+\rho}{1-\rho}} x\right) d x=2 \int_{a}^{+\infty} \Psi\left(\sqrt{\frac{1+\rho}{1-\rho}} x\right) \varphi(x) d x .
$$


Now, using polar coordinates, it is easy to establish that

$$
\int_{0}^{+\infty} \Psi(k x) \varphi(x) d x=\frac{1}{2 \pi} \arctan (k)
$$

which yields the first expression.

Proof of point (ii). Conditionally to $\left(X_{0}=x, X_{t}=u\right), X_{t}^{\prime}$ is Gaussian with:

- mean $m(t)=\frac{r^{\prime}(t)(x-r(t) u)}{1-r^{2}(t)}$,

- variance $\sigma^{2}(t)$ already defined.

It is easy to check that, if $Z$ is a Gaussian random variable with mean $m$ and variance $\sigma^{2}$, then

$$
\mathbb{E}\left(Z^{+}\right)=\sigma \varphi\left(\frac{m}{\sigma}\right)+m \Phi\left(\frac{m}{\sigma}\right) .
$$

These two remarks yield $\overline{\nu_{1}}(u, T)=I_{1}+I_{2}$, with:

- $I_{1}=\int_{0}^{T} d t \int_{u}^{+\infty} \sigma \varphi\left(\frac{r^{\prime}(x-r u)}{\left(1-r^{2}\right) \sigma}\right) p_{0, t}(x, u) d x$

- $I_{2}=\int_{0}^{T} d t \int_{u}^{+\infty} \frac{r^{\prime}(x-r u)}{\left(1-r^{2}\right)} \Phi\left(\frac{r^{\prime}(x-r u)}{\left(1-r^{2}\right) \sigma}\right) p_{0, t}(x, u) d x$.

$I_{1}$ can be written under the following form: $I_{1}=\varphi(u) \int_{0}^{T} \frac{\sigma^{2}}{\sqrt{2 \pi \lambda_{2}}} \bar{\Phi}\left(\frac{\sqrt{\lambda_{2}}}{\sigma} \sqrt{\frac{1-r}{1+r}} u\right) d t$. Integrating $I_{2}$ by parts leads to

$$
I_{2}=\varphi(u) \int_{0}^{T}\left[\begin{array}{c}
\frac{r^{\prime}}{\sqrt{1-r^{2}}} \varphi\left(\sqrt{\frac{1-r}{1+r}} u\right) \bar{\Phi}(b) \\
+\frac{r^{\prime 2}}{\sqrt{2 \pi \lambda_{2}}\left(1-r^{2}\right)} \bar{\Phi}\left(\frac{\sqrt{\lambda_{2}}}{\sigma} \sqrt{\frac{1-r}{1+r}} u\right)
\end{array}\right] d t .
$$

Finally, noticing that $\sigma^{2}+\frac{r^{\prime 2}}{1-r^{2}}=\lambda_{2}$, we obtain:

$$
\overline{\nu_{1}}(u, T)=\sqrt{\frac{\lambda_{2}}{2 \pi}} \varphi(u) \int_{0}^{T} \bar{\Phi}\left(\frac{\sqrt{\lambda_{2}}}{\sigma} \sqrt{\frac{1-r}{1+r}} u\right) d t+\varphi(u) \int_{0}^{T} \frac{r^{\prime}}{\sqrt{1-r^{2}}} \varphi\left(\sqrt{\frac{1-r}{1+r}} u\right) \bar{\Phi}(b) d t .
$$

Proof of point (iii). We set:

- $v(x, y)=\frac{(x-b)^{2}-2 \rho(x-b)(y+b)+(y+b)^{2}}{2\left(1-\rho^{2}\right)}$

- for $(i, j) \in\{(0,0) ;(1,0) ;(0,1) ;(1,1) ;(2,0) ;(0,2)\}$

$$
J_{i j}=\int_{0}^{+\infty} \int_{0}^{+\infty} \frac{x^{i} y^{j}}{2 \pi \sqrt{1-\rho^{2}}} \exp (-v(x, y)) d y d x .
$$

We first calculate the values of $J_{i j}$. The following relation is clear

$$
\begin{aligned}
J_{10}-\rho J_{01}-(1+\rho) b J_{00} & =\left(1-\rho^{2}\right) \int_{0}^{+\infty}\left(\int_{0}^{+\infty} \frac{\partial}{\partial x} v(x, y) \frac{\exp (-v(x, y))}{2 \pi \sqrt{1-\rho^{2}}} d x\right) d y \\
& =\left(1-\rho^{2}\right) \bar{\Phi}(k b) \varphi(b) .
\end{aligned}
$$


Symmetrically, replacing $x$ with $y$ and $b$ with $-b$ in (4.2) yields

$$
J_{01}-\rho J_{10}+(1+\rho) b J_{00}=\left(1-\rho^{2}\right) \Phi(k b) \varphi(b) .
$$

In the same way, multiplying the integrand by $y$, we get

$$
J_{11}-\rho J_{02}-(1+\rho) b J_{01}=\left(1-\rho^{2}\right)^{3 / 2}[\varphi(k b)-k b \bar{\Phi}(k b)] \varphi(b) .
$$

And then, multiplying the integrand by $x$ leads to

$$
J_{11}-\rho J_{20}+(1+\rho) b J_{10}=\left(1-\rho^{2}\right)^{3 / 2}[\varphi(k b)+k b \Phi(k b)] \varphi(b) .
$$

Finally, $J_{20}-\rho J_{11}-(1+\rho) b J_{10}=\left(1-\rho^{2}\right) \int_{0}^{+\infty} \int_{0}^{+\infty} x \frac{\partial}{\partial x} v(x, y) \frac{\exp (-v(x, y))}{2 \pi \sqrt{1-\rho^{2}}} d x d y$. Then, integrating by parts

$$
J_{20}-\rho J_{11}-(1+\rho) b J_{10}=\left(1-\rho^{2}\right) J_{00} .
$$

Multiplying equation (4.6) by $\rho$ and adding (4.5) gives:

$$
J_{11}=-b J_{10}+\rho J_{00}+\sqrt{1-\rho^{2}}[\varphi(k b)+k b \Phi(k b)] \varphi(b) .
$$

Multiplying equation (4.3) by $\rho$ and adding equation (4.2) yields:

$$
J_{10}=b J_{00}+[\Phi(k b)+\rho \Phi(k b)] \varphi(b) .
$$

And, by formula $(i), J_{00}=2 \int_{b}^{+\infty} \Psi(k x) \varphi(x) d x$. Finally, gathering the pieces, it comes:

$$
J_{11}=J_{11}(b, \rho)=\sqrt{1-\rho^{2}} \varphi^{2}\left(\frac{b}{\sqrt{1-\rho}}\right) \varphi(b)+2\left(\rho-b^{2}\right) \int_{b}^{+\infty} \Psi(k x) \varphi(x) d x+2 b \Psi(k b) \varphi(b) .
$$

The final result is obtained remarking that

$$
\mathbb{E}\left(\left(X_{0}^{\prime}\right)^{+}\left(X_{t}^{\prime}\right)^{+} \mid X_{0}=X_{t}=u\right)=\sigma^{2}(t) J_{11}(b(t), \rho(t)) .
$$

Proof of point (iv). Expression (2.4) is obtained simply using the second expression of $J_{00}$.

Note 4.1. In the following proofs, some expansions are made as $t \longrightarrow 0$, some as $u \longrightarrow+\infty$ and some as $(t, u) \longrightarrow(0,+\infty)$.

We define the uniform Landau symbol $O_{U}$ as $a(t, u)=O_{U}(b(t, u))$ if there exists $T_{0}$ and $u_{0}$ such that for $t<T_{0}<T$ and $u>u_{0}$,

$$
a(t, u) \leq \text { const }) b(t, u)
$$

We also define the symbol $\asymp$ as $a(t, u) \asymp b(t, u) \Longleftrightarrow\left\{\begin{array}{l}a(t, u)=O_{U}(b(t, u)) \\ b(t, u)=O_{U}(a(t, u))\end{array}\right.$.

Note 4.2. Many results of this section are based on tedious Taylor expansions. These expansions have been made or checked by a computer algebra system (Maple). They are not detailed in the proofs. 
Proof of Proposition 2.3. Use form (iii) and remark that, when $t$ is small, $k(t)=\sqrt{\frac{1+\rho(t)}{1-\rho(t)}}=O(t)$ is small, and, since $\Psi(\varepsilon)=\frac{1}{\sqrt{2 \pi}}\left(\varepsilon-\frac{\varepsilon^{3}}{6}\right)+O\left(\varepsilon^{5}\right)$ as $\varepsilon \rightarrow 0$, we get:

$$
\begin{gathered}
T_{2}(t)=2\left(\sigma^{2}(t) \rho(t)-\mu^{2}(t)\right)\left[\frac{\arctan (k(t))}{2 \pi}-\frac{k(t)}{\sqrt{2 \pi}} \int_{0}^{b(t)} x \varphi(x) d x+\frac{k^{3}(t)}{6 \sqrt{2 \pi}} \int_{0}^{b(t)} x^{3} \varphi(x) d x\right]+O\left(t^{5}\right) \\
=2\left(\sigma^{2}(t) \rho(t)-\mu^{2}(t)\right)\left[\begin{array}{l}
\frac{1}{2 \pi} \arctan (k(t))-\frac{k(t)}{\sqrt{2 \pi}}(\varphi(0)-\varphi(b(t))) \\
+\frac{k^{3}(t)}{6 \sqrt{2 \pi}}\left(2 \varphi(0)-\left(b^{2}(t)+2\right) \varphi(b(t))\right)
\end{array}\right]+O\left(t^{5}\right) .
\end{gathered}
$$

In the same way:

$$
T_{3}(t)=\frac{2 \mu(t) \sigma(t)}{\sqrt{2 \pi}} \varphi(b(t))\left[k(t) b(t)-\frac{k^{3}(t)}{6} b^{3}(t)\right]+O\left(t^{5}\right) .
$$

And then, assuming $\lambda_{8}$ finite, use Maple to get the result.

Proof of Proposition 2.4. We first prove the following two lemmas.

Lemma 4.3. Let $l$ be a real positive function of class $\mathcal{C}^{2}$ satisfying $l(t)=c t+O\left(t^{2}\right)$ as $t \longrightarrow 0, c>0$. Suppose that $\lambda_{8}$ is finite, with the above definitions of $k(t)$ and $b(t)$, we have as $u \longrightarrow+\infty$ :

$$
\text { (i) } I_{p}=\int_{0}^{T} t^{p} \Psi(k(t) b(t)) \varphi(l(t) u) d t=(c u)^{-(p+1)} \frac{1}{\sqrt{2 \pi}} \frac{M_{p+1}}{2} \int_{0}^{\arctan \left(\frac{d}{c}\right)}(\cos \theta)^{p} d \theta\left[1+O\left(\frac{1}{u}\right)\right]
$$

with $d=\frac{1}{6} \frac{\sqrt{\lambda_{2}^{2} \lambda_{6}-\lambda_{2} \lambda_{4}^{2}}}{\lambda_{4}-\lambda_{2}^{2}}$ and $M_{p+1}=\mathbb{E}\left(|Z|^{p+1}\right)$ where $Z$ is a standard Gaussian random variable.

(ii) $J_{p}=\int_{0}^{T} t^{p} \varphi(l(t) u) d t=(c u)^{-(p+1)} \frac{M_{p}}{2}\left[1+O\left(\frac{1}{u}\right)\right]$.

Proof of Lemma 4.3. Since the derivative of $l$ at zero is non zero, $l$ is invertible in some neighbourghood of zero and its inverse $l^{-1}$ satisfies $l^{-1}(t)=\frac{1}{c} t+O\left(t^{2}\right),\left(l^{-1}\right)^{\prime}(t)=\frac{1}{c}+O(t)$.

We first consider $I_{p}$ and use the change of variable $y=l(t) u$, then

$$
I_{p}=\int_{0}^{l(T) u}\left(l^{-1}\left(\frac{y}{u}\right)\right)^{p} \Psi\left[(k b) \circ l^{-1}\left(\frac{y}{u}\right)\right] \varphi(y)\left(l^{-1}\right)^{\prime}\left(\frac{y}{u}\right) \frac{d y}{u} .
$$

From the expressions of $k(t)$ and $b(t)$, we know that

$$
(k b)(t)=\frac{1}{6} \frac{\sqrt{\lambda_{2}^{2} \lambda_{6}-\lambda_{2} \lambda_{4}^{2}}}{\lambda_{4}-\lambda_{2}^{2}} t u+u O\left(t^{3}\right)=d u t+u O\left(t^{3}\right) .
$$

Thus $(k b) \circ l^{-1}\left(\frac{y}{u}\right)=\frac{d}{c} y+u O_{U}\left(\frac{y^{2}}{u^{2}}\right)$ and

$$
I_{p}=(c u)^{-(p+1)} \int_{0}^{l(T) u} y^{p} \Psi\left(\frac{d}{c} y+u O_{U}\left(\frac{y^{2}}{u^{2}}\right)\right) \varphi(y)\left[1+O_{U}\left(\frac{y}{u}\right)\right] d y .
$$

We use the following lemma. 
Lemma 4.4. Let $h$ be a real function such that $h(t)=O\left(t^{2}\right)$ as $t \searrow 0$, then there exists $T_{0}$ such that for $0 \leq t \leq T_{0}$

$$
\Psi(u(t+h(t)))=\Psi(t u)\left[1+O_{U}(t)\right] .
$$

Proof of Lemma 4.4. Taking $T_{0}$ sufficiently small, we can assume that $h(t) \leq \frac{t}{2}$. Then

$$
A=|\Psi(u(t+h(t)))-\Psi(t u)| \leq u|h(t)| \varphi\left(\frac{t u}{2}\right) \leq(\text { const }) u t^{2} \varphi\left(\frac{t u}{2}\right) .
$$

We want to prove that, in every case,

$$
A \leq(\text { const }) t \Psi(t u)
$$

- when $t u \leq 1, \Psi(t u) \geq t u \varphi(1)$ and $A \leq($ const $) u t^{2} \varphi(0)$, thus (4.7) holds.

- when $t u>1, \Psi(t u)>\Psi(1)$ and $A \leq($ const $) t^{2} u \varphi\left(\frac{u t}{2}\right)$ and (4.7) holds again.

End of proof of Lemma 4.3.

Due to Lemma 4.4,

$$
I_{p}=(c u)^{-(p+1)} \int_{0}^{l(T) u} y^{p} \Psi\left(\frac{d}{c} y\right) \varphi(y)\left[1+O_{U}\left(\frac{y}{u}\right)\right] d y .
$$

Put $K_{p}(u)=\int_{0}^{l(T) u} y^{p} \Psi\left(\frac{d}{c} y\right) \varphi(y) d y$. It is easy to see that, when $u \longrightarrow+\infty$,

$$
K_{p}(u)=\int_{0}^{+\infty} y^{p} \Psi\left(\frac{d}{c} y\right) \varphi(y) d y+O\left(u^{-n}\right) \text { for every integer } n>0 .
$$

Moreover, $K_{p}(\infty)=\int_{0}^{+\infty} y^{p} \Psi\left(\frac{d}{c} y\right) \varphi(y) d y=\int_{0}^{+\infty} \int_{0}^{\frac{d}{c} y} \frac{y^{p}}{2 \pi} \exp -\frac{y^{2}+z^{2}}{2} d z d y$. Then, using polar coordinates, we derive that $K_{p}(\infty)=\frac{1}{\sqrt{2 \pi}} \frac{M_{p+1}}{2} \int_{0}^{\arctan \left(\frac{d}{c}\right)}(\cos \theta)^{p} d \theta$. So we can see that the contribution of the term $O_{U}\left(\frac{y}{u}\right)$ in formula $(4.8)$ is $O\left(u^{-(p+2)}\right)$ which gives the desired result for $I_{p}$.

The same kind of proof gives the expression of $J_{p}$.

Proof of the equivalent of $\bar{\nu}_{1}(u, T)$. We set

$$
A_{1}(t)=\varphi(u)\left(\sqrt{\frac{\lambda_{2}}{2 \pi}} \bar{\Phi}\left(\sqrt{\frac{\lambda_{2}(1-r)}{\sigma^{2}(1+r)}} u\right)+\varphi\left(\sqrt{\frac{1-r}{1+r}} u\right) \Phi(b) \frac{r^{\prime}}{\sqrt{1-r^{2}}}\right) .
$$

Then, $\bar{\nu}_{1}(u, T)=\int_{0}^{T} A_{1}(t) d t$.

It is well known ([1], p. 932) that, as $z$ tends to infinity,

$$
\bar{\Phi}(z)=\varphi(z)\left[\frac{1}{z}-\frac{1}{z^{3}}+\frac{3}{z^{5}}+O\left(z^{-7}\right)\right] .
$$


We use this expansion for both terms of $\bar{\nu}_{1}(u, T)$, with $z=\sqrt{\frac{\lambda_{2}(1-r(t))}{\sigma^{2}(t)(1+r(t))}} u$ for the first term and $z=b(t)$ for the second one.

Besides, remarking that

$$
\varphi\left(\sqrt{\frac{\lambda_{2}(1-r)}{\sigma^{2}(1+r)}} u\right)=\sqrt{2 \pi} \varphi\left(\sqrt{\frac{1-r}{1+r}} u\right) \varphi(b)
$$

we get:

$$
A_{1}(t)=\frac{\varphi(u)}{\sqrt{2 \pi}} \varphi\left(\sqrt{\frac{\lambda_{2}(1-r)}{\sigma^{2}(1+r)} u}\right)\left(\begin{array}{c}
\sqrt{\sqrt{\frac{\sigma^{2}(1+r)}{\lambda_{2}(1-r)}} \frac{1}{u}-\left(\frac{\sigma^{2}(1+r)}{\lambda_{2}(1-r)}\right)^{3 / 2} \frac{1}{u^{3}}} \\
+O_{U}\left(\left(\frac{\sigma^{2}(1+r)}{\lambda_{2}(1-r)}\right)^{5 / 2} \frac{1}{u^{5}}\right)
\end{array}\right]
$$

From Taylor expansion made by Maple assuming $\lambda_{8}$ finite, we know that:

$$
A_{1}(t)=\frac{1}{8} \frac{\left(\lambda_{4}-\lambda_{2}^{2}\right)^{5 / 2} \exp \left(-\frac{u^{2} \lambda_{4}}{2\left(\lambda_{4}-\lambda_{2}^{2}\right)}\right)}{\lambda_{2}^{7 / 2} u^{3} \pi \sqrt{2 \pi}} t^{2}+O\left(t^{4}\right)
$$

To use Lemma 4.3 point (ii) to calculate $\bar{\nu}_{1}(u, T)$, it is necessary to have a Taylor expansion of the coefficient of $u$ in $\varphi\left(\sqrt{\frac{\lambda_{2}(1-r)}{\sigma^{2}(1+r)}} u\right)$. We have $\lim _{t \rightarrow 0} \frac{\lambda_{2}(1-r(t))}{\sigma^{2}(t)(1+r(t))}=\frac{\lambda_{2}^{2}}{\lambda_{4}-\lambda_{2}^{2}}$, therefore, we set:

$$
l(t)=\sqrt{\frac{\lambda_{2}(1-r)}{\sigma^{2}(1+r)}-\frac{\lambda_{2}^{2}}{\lambda_{4}-\lambda_{2}^{2}}} .
$$

From Taylor expansion made by Maple assuming $\lambda_{8}$ finite, we get

$$
l(t)=\frac{1}{6} \frac{\sqrt{2} \sqrt{\lambda_{2}\left(\lambda_{2} \lambda_{6}-\lambda_{4}^{2}\right)}}{\lambda_{4}-\lambda_{2}^{2}} t+O\left(t^{2}\right) .
$$

And, according to Lemma 4.3 point $(i i)$,

$$
\int_{0}^{T} t^{2} \varphi(l(t) u) d t=\frac{1}{2}\left(\frac{1}{6} \frac{\sqrt{2} \sqrt{\lambda_{2}\left(\lambda_{2} \lambda_{6}-\lambda_{4}^{2}\right)}}{\lambda_{4}-\lambda_{2}^{2}} u\right)^{-3}\left(1+O\left(\frac{1}{u}\right)\right) .
$$

Finally, remarking that $\varphi(u) \varphi\left(\frac{\lambda_{2}}{\sqrt{\lambda_{4}-\lambda_{2}^{2}}} u\right)=\frac{1}{\sqrt{2 \pi}} \varphi\left(\sqrt{\frac{\lambda_{4}}{\lambda_{4}-\lambda_{2}^{2}}} u\right)$, we get the equivalent for $\bar{\nu}_{1}(u, T)$.

$$
\bar{\nu}_{1}(u, T)=\frac{27}{4 \sqrt{\pi}} \frac{\left(\lambda_{4}-\lambda_{2}^{2}\right)^{11 / 2}}{\lambda_{2}^{5}\left(\lambda_{2} \lambda_{6}-\lambda_{4}^{2}\right)^{3 / 2}} \varphi\left(\sqrt{\frac{\lambda_{4}}{\lambda_{4}-\lambda_{2}^{2}}} u\right) u^{-6}\left(1+O\left(\frac{1}{u}\right)\right) .
$$


Proof of the equivalent of $\nu_{2}(u, T)$. Remember that

$$
\nu_{2}(u, T)=\int_{0}^{T} 2(T-t) \frac{1}{\sqrt{1-r^{2}(t)}} \varphi^{2}\left(\frac{u}{\sqrt{1+r(t)}}\right)\left[T_{1}(t)+T_{2}(t)+T_{3}(t)\right] d t .
$$

We first calculate an expansion of term $T_{2}=2 \sigma^{2}\left(\rho-b^{2}\right) \int_{b}^{+\infty} \varphi(x) \Psi(k x) d x$.

The function $x \longrightarrow\left(x^{2}-1\right) \varphi(x)$ being bounded, we have

$$
\Psi(k x)=\Psi(k b)+k \varphi(k b)(x-b)-\frac{1}{2} k^{3} b \varphi(k b)(x-b)^{2}+O_{U}\left(k^{3}(x-b)^{3}\right),
$$

where the Landau's symbol has here the same meaning as in Lemma 4.3.

Moreover, using the expansion of $\bar{\Phi}$ given in formula (4.9), it is easy to check that as $z \longrightarrow+\infty$,

$$
\begin{aligned}
& -\int_{z}^{+\infty}(x-z) \varphi(x) d x=\frac{\varphi(z)}{z^{2}}-3 \frac{\varphi(z)}{z^{4}}+O\left(\frac{\varphi(z)}{z^{6}}\right) \\
& \text { - } \int_{z}^{+\infty}(x-z)^{2} \varphi(x) d x=2 \frac{\varphi(z)}{z^{3}}+O\left(\frac{\varphi(z)}{z^{5}}\right) \\
& \text { - } \int_{z}^{+\infty}(x-z)^{3} \varphi(x) d x=O\left(\frac{\varphi(z)}{z^{4}}\right) .
\end{aligned}
$$

Therefore, multiplying formula (4.11) by $\varphi(x)$, integrating on $[b ;+\infty[$ and applying formula (4.9) once again yield:

$$
T_{2}=2 \sigma^{2}\left(\rho-b^{2}\right)\left\{\begin{array}{l}
\Psi(k b) \varphi(b)\left[\frac{1}{b}-\frac{1}{b^{3}}+\frac{3}{b^{5}}\right]+k \varphi(k b) \varphi(b)\left[\frac{1-k^{2}}{b^{2}}-\frac{3}{b^{4}}\right] \\
+O\left(\frac{\Psi(k b) \varphi(b)}{b^{7}}\right)+O\left(\frac{k}{b^{6}} \varphi(k b) \varphi(b)\right) \\
+O\left(\frac{k^{3}}{b^{4}} \varphi(k b) \varphi(b)\right)+O\left(\frac{k^{3}}{b^{4}} \varphi(b)\right)
\end{array}\right\} .
$$

Note that the penultimate term can be forgotten. Then, remarking that, as $u \longrightarrow+\infty, b=\frac{\mu}{\sigma} \asymp u, \sigma \asymp t$ and $k \asymp t$, we obtain:

$$
\begin{aligned}
T_{2}= & -2 \sigma^{2} b \Psi(k b) \varphi(b)+2 \frac{\sigma^{2}}{b} \Psi(k b) \varphi(b)+2 \frac{\sigma^{2} \rho}{b} \Psi(k b) \varphi(b) \\
& -2 \frac{\sigma^{2} \rho}{b^{3}} \Psi(k b) \varphi(b)-6 \frac{\sigma^{2}}{b^{3}} \Psi(k b) \varphi(b)+2 \sigma^{2} k^{3} \varphi(k b) \varphi(b) \\
& -2 \sigma^{2} k \varphi(k b) \varphi(b)+2 \frac{\sigma^{2} \rho k}{b^{2}} \varphi(k b) \varphi(b)+6 \frac{\sigma^{2} k}{b^{2}} \varphi(k b) \varphi(b) \\
& +O_{U}\left(t^{2} u^{-5} \Psi(k b) \varphi(b)\right)+O_{U}\left(t^{3} u^{-4} \varphi(k b) \varphi(b)\right)+O_{U}\left(t^{5} u^{-2} \varphi(b)\right) .
\end{aligned}
$$

Remark 4.5. As it will be seen later on, Lemma 4.3 shows that the contribution of the remainder to the integral (4.10) can be neglected since the degrees in $t$ and $\frac{1}{u}$ of each term are greater than 5 . So, in the sequel, we will denote the sum of these terms (and other terms that will appear later) by Remainder and we set:

$$
T_{2}=U_{1}+U_{2}+U_{3}+U_{4}+U_{5}+U_{6}+U_{7}+U_{8}+U_{9}+\text { Remainder }
$$


Now, we have

- $U_{1}+T_{3}=0$

- $\sqrt{1-\rho^{2}}-2 k=-(1+\rho) k$ so that $U_{7}+T_{1}=-(1+\rho) \sigma^{2} k \varphi(k b) \varphi(b)$

- $U_{2}+U_{3}=2 \frac{\sigma^{2}}{b}(1+\rho) \Psi(k b) \varphi(b)$

- $U_{4}+U_{5}=-4 \frac{\sigma^{2}}{b^{3}} \Psi(k b) \varphi(b)\left(1+O\left(t^{2}\right)\right)$

- $U_{8}+U_{9}=4 \frac{\sigma^{2}}{b^{2}} k \varphi(k b) \varphi(b)\left(1+O\left(t^{2}\right)\right)$

since $\rho=-1+O\left(t^{2}\right)$.

By the same remark as Remark 4.5 above, the term $O\left(t^{2}\right)$ can be neglected. Consequently,

$$
\begin{aligned}
T_{1}+T_{2}+T_{3}= & 2 \frac{\sigma^{2}}{b}(1+\rho) \Psi(k b) \varphi(b)-4 \frac{\sigma^{2}}{b^{3}} \Psi(k b) \varphi(b) \\
& -(1+\rho) \sigma^{2} k \varphi(k b) \varphi(b)+2 \sigma^{2} k^{3} \varphi(k b) \varphi(b)+4 \frac{\sigma^{2}}{b^{2}} k \varphi(k b) \varphi(b) \\
& + \text { Remainder. }
\end{aligned}
$$

Therefore, we are leaded to use Lemma 4.3 in order to calculate the following integrals:

- $\int_{0}^{T}(T-t) m_{1}(t) \exp \left(-\frac{u^{2}}{1+r}\right) \Psi(k b) \varphi(b) d t=\int_{0}^{T}(T-t) m_{1}(t) \Psi(k b) \varphi\left(\sqrt{b^{2}+\frac{2 u^{2}}{1+r}}\right) d t$

- $\int_{0}^{T}(T-t) m_{2}(t) \Psi(k b) \varphi\left(\sqrt{b^{2}+\frac{2 u^{2}}{1+r}}\right) d t$

- $\int_{0}^{T}(T-t) m_{3}(t) \varphi\left(\sqrt{b^{2}\left(1+k^{2}\right)+\frac{2 u^{2}}{1+r}}\right) d t$

- $\int_{0}^{T}(T-t) m_{4}(t) \varphi\left(\sqrt{b^{2}\left(1+k^{2}\right)+\frac{2 u^{2}}{1+r}}\right) d t$

- $\int_{0}^{T}(T-t) m_{5}(t) \varphi\left(\sqrt{b^{2}\left(1+k^{2}\right)+\frac{2 u^{2}}{1+r}}\right) d t$ 
with:

$$
\begin{aligned}
m_{1}(t) & =\frac{2}{\pi} \frac{1}{\sqrt{1-r^{2}(t)}} \frac{\sigma^{2}}{b}(t)(1+\rho(t)) \\
& =\frac{1}{36} \frac{\left(\lambda_{2} \lambda_{6}-\lambda_{4}^{2}\right) \sqrt{\lambda_{4}-\lambda_{2}^{2}}}{\pi \lambda_{2}^{5 / 2} u} t^{3}+O\left(t^{5}\right) \\
\text { - } m_{2}(t) & =-\frac{4}{\pi} \frac{1}{\sqrt{1-r^{2}(t)}} \frac{\sigma^{2}}{b^{3}}(t)=-\frac{\left(\lambda_{4}-\lambda_{2}^{2}\right)^{5 / 2}}{\pi u^{3} \lambda_{2}^{7 / 2}} t+O\left(t^{3}\right) \\
\text { - } m_{3}(t) & =-\frac{1}{\pi \sqrt{2 \pi}} \frac{1}{\sqrt{1-r^{2}(t)}}(1+\rho(t)) \sigma^{2}(t) k(t) \\
& =-\frac{\sqrt{2}}{864} \frac{\left(\lambda_{2} \lambda_{6}-\lambda_{4}^{2}\right)^{3 / 2}}{\lambda_{2}^{2} \sqrt{\lambda_{4}-\lambda_{2}^{2}} \pi^{3 / 2}} t^{4}+O\left(t^{6}\right) \\
\text { - } m_{4}(t) & =\frac{2}{\pi \sqrt{2 \pi}} \frac{1}{\sqrt{1-r^{2}(t)}} \sigma^{2}(t) k^{3}(t) \\
& =\frac{1}{864} \frac{\left(\lambda_{2} \lambda_{6}-\lambda_{4}^{2}\right)^{3 / 2}}{\lambda_{2}^{2} \sqrt{\lambda_{4}-\lambda_{2}^{2}} \pi^{3 / 2}} t^{4}+O\left(t^{6}\right) \\
-m_{5}(t) & =\frac{4}{\pi \sqrt{2 \pi}} \frac{1}{\sqrt{1-r^{2}(t)}} \frac{\left.\sigma^{(} 1998\right) .2}{b^{2}}(t) k(t) \\
& =\frac{1}{12} \frac{\sqrt{\lambda_{2} \lambda_{6}-\lambda_{4}^{2}}\left(\lambda_{4}-\lambda_{2}^{2}\right)^{3 / 2} \sqrt{2}}{\lambda_{2}^{3} \pi^{3 / 2} u^{2}} t^{2}+O\left(t^{4}\right) .
\end{aligned}
$$

Lemma 4.3 shows that we can neglect the terms issued from the $t$ part of the factor $T-t$ in formula (4.10).

We now consider the argument of $\varphi$ in Lemma 4.3. We have:

- $\lim _{t \rightarrow 0} \frac{b^{2}}{u^{2}}+\frac{2}{1+r}=\frac{\lambda_{4}}{\lambda_{4}-\lambda_{2}^{2}}$

- $\lim _{t \rightarrow 0} \frac{b^{2}}{u^{2}}\left(1+k^{2}\right)+\frac{2}{1+r}=\frac{\lambda_{4}}{\lambda_{4}-\lambda_{2}^{2}}$.

Therefore, we set:

$$
\begin{aligned}
-l_{1}(t) & =\sqrt{\frac{b^{2}(t)}{u^{2}}+\frac{2}{1+r(t)}-\frac{\lambda_{4}}{\lambda_{4}-\lambda_{2}^{2}}}=\sqrt{\frac{\lambda_{2}\left(\lambda_{2} \lambda_{6}-\lambda_{4}^{2}\right)}{18\left(\lambda_{4}-\lambda_{2}^{2}\right)^{2}}} t+O\left(t^{3}\right) \\
\text { - } l_{2}(t) & =\sqrt{\frac{b^{2}(t)}{u^{2}}\left(1+k^{2}(t)\right)+\frac{2}{1+r}-\frac{\lambda_{4}}{\lambda_{4}-\lambda_{2}^{2}}} \\
& =\sqrt{\frac{\lambda_{2}\left(\lambda_{2} \lambda_{6}-\lambda_{4}^{2}\right)}{12\left(\lambda_{4}-\lambda_{2}^{2}\right)^{2}}} t+O\left(t^{3}\right) .
\end{aligned}
$$

Then, with the notations of Lemma 4.3, we obtain:

$$
\nu_{2}=T \exp \left(-\frac{\lambda_{4} u^{2}}{2\left(\lambda_{4}-\lambda_{2}^{2}\right)}\right)\left[\begin{array}{l}
\frac{1}{36} \frac{\left(\lambda_{2} \lambda_{6}-\lambda_{4}^{2}\right) \sqrt{\lambda_{4}-\lambda_{2}^{2}}}{\pi \lambda_{2}^{5 / 2} u} I_{3}-\frac{\left(\lambda_{4}-\lambda_{2}^{2}\right)^{5 / 2}}{\pi u^{3} \lambda_{2}^{7 / 2}} I_{1} \\
+\frac{1}{12} \frac{\sqrt{\lambda_{2} \lambda_{6}-\lambda_{4}^{2}}\left(\lambda_{4}-\lambda_{2}^{2}\right)^{3 / 2} \sqrt{2}}{\lambda_{2}^{3} \pi^{3 / 2} u^{2}} J_{2}
\end{array}\right]\left(1+O\left(\frac{1}{u}\right)\right)
$$


Where $I_{1}$ and $I_{3}$ (resp. $\left.J_{2}\right)$ are defined as in Lemma 4.3 point $(i)($ resp. $(i i))$ with $l(t)=l_{1}(t)\left(\right.$ resp. $\left.l(t)=l_{2}(t)\right)$. Noting that $\int_{0}^{\frac{\sqrt{2}}{2}}(\cos \theta)^{3} d \theta=\frac{8 \sqrt{3}}{27}$ and that $\int_{0}^{\frac{\sqrt{2}}{2}} \cos \theta d \theta=\frac{\sqrt{3}}{3}$, we find

- $I_{3}=\frac{144 \sqrt{3}\left(\lambda_{4}-\lambda_{2}^{2}\right)^{4}}{\sqrt{2 \pi} \lambda_{2}^{2}\left(\lambda_{2} \lambda_{6}-\lambda_{4}^{2}\right)^{2}} u^{-4}\left(1+O\left(\frac{1}{u}\right)\right)$

- $I_{1}=\frac{3 \sqrt{3}\left(\lambda_{4}-\lambda_{2}^{2}\right)^{2}}{\sqrt{2 \pi} \lambda_{2}\left(\lambda_{2} \lambda_{6}-\lambda_{4}^{2}\right)} u^{-2}\left(1+O\left(\frac{1}{u}\right)\right)$

- $J_{2}=\frac{12 \sqrt{3}\left(\lambda_{4}-\lambda_{2}^{2}\right)^{3}}{\lambda_{2}\left(\lambda_{2} \lambda_{6}-\lambda_{4}^{2}\right) \sqrt{\lambda_{2}\left(\lambda_{2} \lambda_{6}-\lambda_{4}^{2}\right)}} u^{-3}\left(1+O\left(\frac{1}{u}\right)\right)$.

Finally, gathering the pieces, we obtain the desired expression of $\nu_{2}$.

\section{Discussion}

Using the general relation (1.3) with $n=1$, we get

$$
\left|P\left(X^{\star} \geq u\right)-P\left(X_{0}>u\right)-\tilde{\nu}_{1}(u, T)+\frac{\nu_{2}(u, T)}{2}\right| \leq \frac{\bar{\nu}_{2}(u, T)}{2}+\frac{\nu_{3}(u, T)}{6} .
$$

A conjecture is that the orders of magnitude of $\bar{\nu}_{2}(u, T)$ and $\nu_{3}(u, T)$ are considerably smaller than those of $\bar{\nu}_{1}(u, T)$ and $\nu_{2}(u, T)$. Admitting this conjecture, Proposition 2.4 implies that for $T$ small enough

$$
P\left(X^{\star} \geq u\right)=\bar{\Phi}(u)+\frac{T \sqrt{\lambda_{2}}}{\sqrt{2 \pi}} \varphi(u)-\frac{3 \sqrt{3} T}{2 \pi} \frac{\left(\lambda_{4}-\lambda_{2}^{2}\right)^{9 / 2}}{\lambda_{2}^{9 / 2}\left(\lambda_{2} \lambda_{6}-\lambda_{4}^{2}\right)} \varphi\left(\sqrt{\frac{\lambda_{4}}{\lambda_{4}-\lambda_{2}^{2}}} u\right) u^{-5}\left(1+O\left(\frac{1}{u}\right)\right)
$$

which is Piterbarg's theorem with a better remainder ([15], Th. 3.1, p. 703). Piterbarg's theorem is, as far as we know, the most precise expansion of the distribution of the maximum of smooth Gaussian processes. Moreover, very tedious calculations would give extra terms of the Taylor expansion.

\section{REFERENCES}

[1] M. Abramowitz and I.A. Stegun, Handbook of Mathematical Functions with Formulas, Graphs and Mathematical Tables. Dover, New York (1972).

[2] R.J. Adler, An Introduction to Continuity, Extrema and Related Topics for General Gaussian Processes, IMS, Hayward, Ca (1990).

[3] J.-M. Azaïs and M. Wschebor, Une formule pour calculer la distribution du maximum d'un processus stochastique. C.R. Acad. Sci. Paris Ser. I Math. 324 (1997) 225-230.

[4] J-M. Azaïs and M. Wschebor, The Distribution of the Maximum of a Stochastic Process and the Rice Method, submitted.

[5] C. Cierco, Problèmes statistiques liés à la détection et à la localisation d'un gène à effet quantitatif. PHD dissertation. University of Toulouse, France (1996).

[6] C. Cierco and J.-M. Azaïs, Testing for Quantitative Gene Detection in Dense Map, submitted.

[7] H. Cramér and M.R. Leadbetter, Stationary and Related Stochastic Processes, J. Wiley \& Sons, New-York (1967).

[8] D. Dacunha-Castelle and E. Gassiat, Testing in locally conic models, and application to mixture models. ESAIM: Probab. Statist. 1 (1997) 285-317.

[9] R.B. Davies, Hypothesis testing when a nuisance parameter is present only under the alternative. Biometrika 64 (1977) $247-254$.

[10] J. Ghosh and P. Sen, On the asymptotic performance of the log-likelihood ratio statistic for the mixture model and related results, in Proc. of the Berkeley conference in honor of Jerzy Neyman and Jack Kiefer, Le Cam L.M. and Olshen R.A., Eds. (1985). 
[11] M.F. Kratz and H. Rootzén, On the rate of convergence for extreme of mean square differentiable stationary normal processes. J. Appl. Prob. 34 (1997) 908-923.

[12] M.R. Leadbetter, G. Lindgren and H. Rootzén, Extremes and Related Properties of Random Sequences and Processes. SpringerVerlag, New-York (1983).

[13] R.N. Miroshin, Rice series in the theory of random functions. Vestnik Leningrad Univ. Math. 1 (1974) 143-155.

[14] M.B. Monagan, et al. Maple V Programming guide. Springer (1998).

[15] V.I. Piterbarg, Comparison of distribution functions of maxima of Gaussian processes. Theory Probab. Appl. 26 (1981) 687-705.

[16] V.I. Piterbarg, Large deviations of random processes close to gaussian ones. Theory Probab. Appl. 27 (1982) $504-524$.

[17] V.I. Piterbarg, Asymptotic Methods in the Theory of Gaussian Processes and Fields. American Mathematical Society. Providence, Rhode Island (1996).

[18] S.O. Rice, Mathematical Analysis of Random Noise. Bell System Tech. J. 23 (1944) 282-332; 24 (1945) 45-156.

[19] SPLUS, Statistical Sciences, S-Plus Programmer's Manual, Version 3.2, Seattle: StatSci, a division of MathSoft, Inc. (1993).

[20] J. Sun, Significance levels in exploratory projection pursuit. Biometrika 78 (1991) 759-769.

[21] M. Wschebor, Surfaces aléatoires. Mesure géometrique des ensembles de niveau. Springer-Verlag, New-York, Lecture Notes in Mathematics 1147 (1985). 


\section{TAYLOR EXPANSIONS BY MAPLE}

GENERAL FORMULAE

$>\operatorname{phi}:=\mathrm{t}->\exp (-\mathrm{t} * \mathrm{t} / 2) / \operatorname{sqrt}(2 * \mathrm{pi})$;

$$
\phi:=t \rightarrow \frac{e^{\left(-1 / 2 t^{2}\right)}}{\sqrt{2 \pi}}
$$

We introduce mu4=lambda4-lambda $2 \wedge 2$ and mu6 $=$ lambda $2 * 1$ ambda6 -1 ambda $4{ }^{\wedge} 2$ to make the outputs clearer.

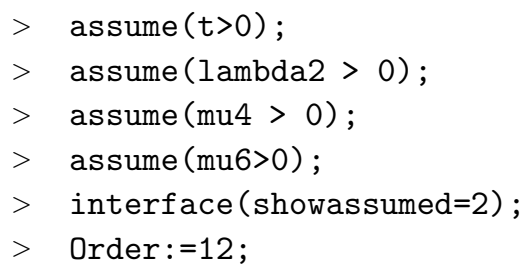

Order $:=12$

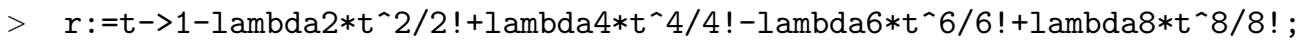

$$
r:=t \rightarrow 1-\frac{1}{2} \lambda 2 t^{2}+\frac{1}{24} \lambda 4 t^{4}-\frac{1}{720} \lambda 6 t^{6}+\frac{1}{40320} \lambda 8 t^{8}
$$

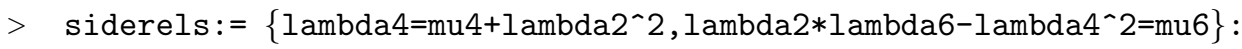

$>\quad I_{-} r 2:=t->1-r(t) * r(t)$;

$$
I \_r 2:=t \rightarrow 1-\mathrm{r}(t)^{2}
$$

$>$ simplify(simplify(series(I_r2(t), t=0,8), siderels));

$$
\lambda 2 t^{2}+\left(-\frac{1}{3} \lambda 2^{2}-\frac{1}{12} \mu 4\right) t^{4}+\left(\frac{1}{360} \lambda 6+\frac{1}{24} \lambda 2 \mu 4+\frac{1}{24} \lambda 2^{3}\right) t^{6}+\mathrm{O}\left(t^{8}\right)
$$

with assumptions on $t, \lambda 2$ and $\mu 4$

$>\quad r p:=t->\operatorname{diff}(r(t), t)$;

$>\operatorname{eval}(\operatorname{rp}(t))$;

$$
r p:=t \rightarrow \operatorname{diff}(\mathrm{r}(t), t)
$$

$$
-\lambda 2 t+\frac{1}{6} \lambda 4 t^{3}-\frac{1}{120} \lambda 6 t^{5}+\frac{1}{5040} \lambda 8 t^{7}
$$

with assumptions on $\lambda 2$ and $t$

$>\quad r s:=t->\operatorname{diff}(r(t), t \$ 2)$;

$>\operatorname{eval}(r s(t))$;

$$
r s:=t \rightarrow \frac{\partial^{2}}{\partial t^{2}} \mathrm{r}(t)
$$

$$
\begin{gathered}
-\lambda 2+\frac{1}{2} \lambda 4 t^{2}-\frac{1}{24} \lambda 6 t^{4}+\frac{1}{720} \lambda 8 t^{6} \\
\text { with assumptions on } \lambda 2 \text { and } t
\end{gathered}
$$


$>\mathrm{mu}:=\mathrm{t}->-\mathrm{u} * \mathrm{rp}(\mathrm{t}) /(1+\mathrm{r}(\mathrm{t}))$;

$$
\mu:=t \rightarrow-\frac{u \operatorname{rp}(t)}{1+\mathrm{r}(t)}
$$

$>\quad$ sig2:=t->lambda2-rp $(t) * r p(t) / I_{-} r 2(t)$;

$$
\text { sig2 }:=t \rightarrow \lambda 2-\frac{\operatorname{rp}(t)^{2}}{\text { I_r2(t) }}
$$

$>$ simplify(taylor (sig2(t), t=0,8), siderels);

$$
\frac{1}{4} \mu 4 t^{2}+\frac{1}{144} \frac{6 \lambda 2^{2} \mu 4-3 \mu 4^{2}-2 \mu 6}{\lambda 2} t^{4}+\mathrm{O}\left(t^{6}\right)
$$
with assumptions on $t, \mu 4, \lambda 2$ and $\mu 6$

$>\operatorname{sigma}:=t->\operatorname{sqrt}(\operatorname{sig} 2(\mathrm{t}))$;

$$
\sigma:=t \rightarrow \sqrt{\operatorname{sig} 2(t)}
$$

$>$ simplify(taylor $(\operatorname{sigma}(t), t=0,6)$, siderels);

$$
\frac{1}{2} \sqrt{\mu 4} t+\frac{1}{144} \frac{6 \lambda 2^{2} \mu 4-3 \mu 4^{2}-2 \mu 6}{\sqrt{\mu 4} \lambda 2} t^{3}+\mathrm{O}\left(t^{5}\right)
$$

with assumptions on $t, \mu 4, \lambda 2$ and $\mu 6$

$>\quad b:=t->m u(t) / \operatorname{sigma}(t)$;

$$
b:=t \rightarrow \frac{\mu(t)}{\sigma(t)}
$$

$>$ simplify(taylor $(b(t), t=0,6)$, siderels);

$$
\frac{u \lambda 2}{\sqrt{\mu 4}}+\left(-\frac{1}{8} u \sqrt{\mu 4}+\frac{1}{36} \frac{u \mu 6}{\mu 4^{(3 / 2)}}\right) t^{2}+\mathrm{O}\left(t^{4}\right)
$$

with assumptions on $\lambda 2, \mu 4, t$ and $\mu 6$

$>\quad$ sig2rho: =t $->-r s(t)-r(t) * r p(t) * r p(t) / I \_r 2(t)$;

$$
\text { sig2rho }:=t \rightarrow-\mathrm{rs}(t)-\frac{\mathrm{r}(t) \mathrm{rp}(t)^{2}}{\mathrm{I} \_\mathrm{r} 2(t)}
$$

$>$ simplify(taylor ( $\operatorname{sig} 2 r h o(t), t=0,8)$, siderels);

$$
-\frac{1}{4} \mu 4 t^{2}+\frac{1}{144} \frac{-6 \lambda 2^{2} \mu 4+3 \mu 4^{2}+4 \mu 6}{\lambda 2} t^{4}+\mathrm{O}\left(t^{6}\right)
$$

with assumptions on $t, \mu 4, \lambda 2$ and $\mu 6$

$>$ rho:=t->sig2rho(t)/sig2(t);

$$
\rho:=t \rightarrow \frac{\operatorname{sig} 2 \mathrm{rho}(t)}{\operatorname{sig} 2(t)}
$$

$>$ simplify(taylor $(r h o(t), t=0,8)$, siderels $)$;

$$
-1+\frac{1}{18} \frac{\mu 6}{\lambda 2 \mu 4} t^{2}+\mathrm{O}\left(t^{4}\right)
$$

$$
\text { with assumptions on } t, \mu 6, \lambda 2 \text { and } \mu 4
$$




\section{PROOF OF PROPOSITION 2.3}

$>\mathrm{k} 2:=\mathrm{t}->(1+r h o(t)) /(1-r h o(t)) ;$

$$
k 2:=t \rightarrow \frac{1+\rho(t)}{1-\rho(t)}
$$

$>$ sk2:=simplify (taylor $(\mathrm{k} 2(\mathrm{t}), \mathrm{t}=0)$, siderels);

$$
\begin{aligned}
\text { sk2 }: & =\frac{1}{36} \frac{\mu 6}{\lambda 2 \mu 4} t^{2}+\frac{1}{2160}\left(3 \lambda 2^{6} \mu 4+9 \lambda 2^{4} \mu 4^{2}+9 \lambda 2^{2} \mu 4^{3}-2 \mu 6 \lambda 2^{2} \mu 4-3 \lambda 8 \lambda 2^{2} \mu 4\right. \\
& \left.+3 \mu 4^{4}+13 \mu 6 \mu 4^{2}+5 \mu 6^{2}\right) /\left(\lambda 2^{2} \mu 4^{2}\right) t^{4}+\frac{1}{907200}\left(-147 \lambda 2^{8} \mu 4^{2}\right. \\
& +175 \mu 6 \lambda 2^{6} \mu 4-273 \lambda 2^{6} \mu 4^{3}+63 \lambda 2^{4} \mu 4^{4}+196 \mu 6 \lambda 2^{4} \mu 4^{2}+120 \lambda 8 \lambda 2^{4} \mu 4^{2} \\
& +357 \lambda 2^{2} \mu 4^{5}+707 \mu 6 \lambda 2^{2} \mu 4^{3}-195 \lambda 8 \lambda 2^{2} \mu 4^{3}-175 \lambda 8 \lambda 2^{2} \mu 6 \mu 4+168 \mu 4^{6} \\
& \left.+518 \mu 6^{2} \mu 4^{2}+686 \mu 6 \mu 4^{4}+175 \mu 6^{3}\right) /\left(\lambda 2^{3} \mu 4^{3}\right) t^{6}+\mathrm{O}\left(t^{8}\right)
\end{aligned}
$$

with assumptions on $t, \mu 6, \lambda 2$ and $\mu 4$

$>\mathrm{k}:=\mathrm{t}->$ taylor $(\operatorname{sqrt}(\mathrm{sk} 2), \mathrm{t}=0)$;

$$
k:=t \rightarrow \operatorname{taylor}\left(\sqrt{s k^{2}}, t=0\right)
$$

$>$ simplify(taylor $(k(t), t=0,3)$, siderels);

$$
\frac{1}{6} \sqrt{\frac{\mu 6}{\lambda 2 \mu 4}} t+\mathrm{O}\left(t^{3}\right)
$$

with assumptions on $t, \mu 6, \lambda 2$ and $\mu 4$

$>$ sqrtI_rho2:=t->k(t)*(1-rho $(t))$;

$$
\text { sqrtI_rho2 }:=t \rightarrow \mathrm{k}(t)(1-\rho(t))
$$

$>\quad \mathrm{T} 1:=\mathrm{t}->\operatorname{sig} 2(\mathrm{t}) * \operatorname{sqr} t I_{\_} r \mathrm{rho} 2(\mathrm{t}) * \operatorname{phi}(\mathrm{b}(\mathrm{t})) * \operatorname{phi}(\mathrm{k}(\mathrm{t}) * \mathrm{~b}(\mathrm{t}))$;

$$
T 1:=t \rightarrow \operatorname{sig} 2(t) \text { sqrtI_rho2 }(t) \phi(\mathrm{b}(t)) \phi(\mathrm{k}(t) \mathrm{b}(t))
$$

$>$ simplify $(\operatorname{simplify}(\operatorname{series}(\mathrm{T} 1(\mathrm{t}), \mathrm{t}=0,6)$, siderels $)$, power);

$$
\begin{gathered}
\frac{1}{24} \frac{\sqrt{\mu 6} \sqrt{\mu 4} e^{\left(-1 / 2 \frac{u^{2} \lambda 2^{2}}{\mu 4}\right)}}{\sqrt{\lambda 2} \pi} t^{3}-\frac{1}{2880}\left(\left(5 \mu 6^{2} \lambda 2^{2} u^{2}+3 \lambda 2^{2} \mu 4^{2} \lambda 8-3 \lambda 2^{6} \mu 4^{2}-9 \lambda 2^{4} \mu 4^{3}\right.\right. \\
\left.-9 \lambda 2^{2} \mu 4^{4}-15 \mu 6 \lambda 2^{2} \mu 4^{2} u^{2}-18 \mu 6 \lambda 2^{2} \mu 4^{2}-3 \mu 4^{5}+5 \mu 6^{2} \mu 4-3 \mu 6 \mu 4^{3}\right) \\
\left.e^{\left(-1 / 2 \frac{u^{2} \lambda 2^{2}}{\mu 4}\right)}\right) /\left(\sqrt{\mu 6} \mu 4^{(3 / 2)} \lambda 2^{(3 / 2)} \pi\right) t^{5}+\mathrm{O}\left(t^{7}\right)
\end{gathered}
$$

with assumptions on $t, \mu 6, \mu 4$ and $\lambda 2$

$>\mathrm{T} 2:=\mathrm{t}->2 * \operatorname{sig} 2(\mathrm{t}) *\left(\mathrm{rho}(\mathrm{t})-(\mathrm{b}(\mathrm{t}))^{\wedge} 2\right) *(\arctan (\mathrm{k}(\mathrm{t})) /(2 * \mathrm{pi})$

$\left.>\quad-k(t) / \operatorname{sqrt}(2 * \mathrm{pi}) *\left(\operatorname{phi}(0)-\operatorname{phi}(\mathrm{b}(\mathrm{t}))-\mathrm{k}(\mathrm{t})^{\wedge} 2 / 6 *\left(2 * \operatorname{phi}(0)-\left((\mathrm{b}(\mathrm{t}))^{\wedge} 2+2\right) * \mathrm{phi}(\mathrm{b}(\mathrm{t}))\right)\right)\right)$;

$$
\begin{aligned}
& \text { T2 }:=t \rightarrow 2 \operatorname{sig} 2(t)\left(\rho(t)-\mathrm{b}(t)^{2}\right) \\
& \left.\qquad \frac{1}{2} \frac{\arctan (\mathrm{k}(t))}{\pi}-\frac{\mathrm{k}(t)\left(\phi(0)-\phi(\mathrm{b}(t))-\frac{1}{6} \mathrm{k}(t)^{2}\left(2 \phi(0)-\left(\mathrm{b}(t)^{2}+2\right) \phi(\mathrm{b}(t))\right)\right)}{\sqrt{2 \pi}}\right)
\end{aligned}
$$


$>$ simplify(simplify(series $(T 2(t), t=0,6)$, siderels), power);

$$
-\frac{1}{24} \frac{\sqrt{\mu 6}\left(u^{2} \lambda 2^{2}+\mu 4\right) e^{\left(-1 / 2 \frac{u^{2} \lambda 2^{2}}{\mu 4}\right)}}{\sqrt{\mu 4} \sqrt{\lambda 2} \pi} t^{3}+\mathrm{O}\left(t^{5}\right)
$$

with assumptions on $t, \mu 6, \lambda 2$ and $\mu 4$

$>\quad$ T3: $=\mathrm{t}->\left(2 * \operatorname{sig} 2(\mathrm{t}) *\left(\mathrm{k}(\mathrm{t}) * \mathrm{~b}(\mathrm{t})^{\wedge} 2\right)\right) / \operatorname{sqrt}(2 * \mathrm{pi}) *\left(1-(\mathrm{k}(\mathrm{t}) * \mathrm{~b}(\mathrm{t}))^{\wedge} 2 / 6\right) * \operatorname{phi}(\mathrm{b}(\mathrm{t}))$;

$$
\text { T3 }:=t \rightarrow 2 \frac{\operatorname{sig} 2(t) \mathrm{k}(t) \mathrm{b}(t)^{2}\left(1-\frac{1}{6} \mathrm{k}(t)^{2} \mathrm{~b}(t)^{2}\right) \phi(\mathrm{b}(t))}{\sqrt{2 \pi}}
$$

$>$ simplify (simplify (series $(\mathrm{T} 3(t), t=0,6)$, siderels), power);

$$
\begin{aligned}
\frac{1}{24} & \frac{e^{\left(-1 / 2 \frac{u^{2} \lambda 2^{2}}{\mu 4}\right)} \sqrt{\mu 6} \lambda 2^{(3 / 2)} u^{2}}{\sqrt{\mu 4} \pi} t^{3}-\frac{1}{25920} \sqrt{\lambda 2} u^{2}\left(27 \lambda 8 \lambda 2^{2} \mu 4^{2}+35 \mu 6^{2} \lambda 2^{2} u^{2}\right. \\
& -27 \lambda 2^{6} \mu 4^{2}-81 \lambda 2^{4} \mu 4^{3}-81 \lambda 2^{2} \mu 4^{4}-162 \mu 6 \lambda 2^{2} \mu 4^{2}-135 \mu 6 \lambda 2^{2} \mu 4^{2} u^{2} \\
& \left.-27 \mu 4^{5}-45 \mu 6^{2} \mu 4+243 \mu 6 \mu 4^{3}\right) e^{\left(-1 / 2 \frac{u^{2} \lambda 2^{2}}{\mu 4}\right)} /\left(\sqrt{\mu 6} \mu 4^{(5 / 2)} \pi\right) t^{5}+\mathrm{O}\left(t^{7}\right)
\end{aligned}
$$

with assumptions on $t, \lambda 2, \mu 4$ and $\mu 6$

$>A:=t->\left((\operatorname{phi}(u / \operatorname{sqrt}((1+r(t))))) \wedge 2 / \operatorname{sqrt}\left(I_{-} r 2(t)\right)\right) *(T 1(t)+T 2(t)+T 3(t))$;

$$
A:=t \rightarrow \frac{\phi\left(\frac{u}{\sqrt{1+\mathrm{r}(t)}}\right)^{2}(\mathrm{~T} 1(t)+\mathrm{T} 2(t)+\mathrm{T} 3(t))}{\sqrt{\mathrm{I} \_\mathrm{r} 2(t)}}
$$

$>\operatorname{simplify}(\operatorname{simplify}(\operatorname{series}(A(t), t=0,6)$, siderels), power);

$$
\mathrm{O}\left(t^{4}\right)
$$

with assumptions on $t$

PROOF OF THE EQUIVALENT OF NU1

$>\operatorname{Cphib}:=t->\operatorname{phi}(\mathrm{t}) / \mathrm{t}-\mathrm{phi}(\mathrm{t}) / \mathrm{t} \uparrow 3$;

$$
C p h i b:=t \rightarrow \frac{\phi(t)}{t}-\frac{\phi(t)}{t^{3}}
$$

$>\mathrm{sq}:=\mathrm{t}->\operatorname{sqrt}((1-r(\mathrm{t})) /(1+r(\mathrm{t})))$;

$$
s q:=t \rightarrow \sqrt{\frac{1-\mathrm{r}(t)}{1+\mathrm{r}(t)}}
$$

$>$ simplify (simplify( $\operatorname{series}(\mathrm{sq}(\mathrm{t}), \mathrm{t}=0,4)$, siderels), power);

$$
\frac{1}{2} \sqrt{\lambda 2} t-\frac{1}{48} \frac{-2 \lambda 2^{2}+\mu 4}{\sqrt{\lambda 2}} t^{3}+\mathrm{O}\left(t^{5}\right)
$$

with assumptions on $t, \lambda 2$ and $\mu 4$

$>$ nsigma: $=$ t->sigma(t)/sqrt (lambda2);

$$
n \text { sigma }:=t \rightarrow \frac{\sigma(t)}{\sqrt{\lambda 2}}
$$


$>\quad A 1:=t->(1 / \operatorname{sqrt}(2 * \mathrm{pi})) * \operatorname{phi}(\mathrm{u}) * \operatorname{phi}(\mathrm{sq}(\mathrm{t}) * \mathrm{u} / \mathrm{nsigma}(\mathrm{t})) *((\mathrm{nsigma}(\mathrm{t}) /(\mathrm{sq}(\mathrm{t}) * \mathrm{u})$

$\left.\left.>\quad-(\mathrm{nsigma}(\mathrm{t}) /(\mathrm{sq}(\mathrm{t}) * \mathrm{u}))^{\wedge} 3\right) * \operatorname{sqrt}(\operatorname{lambda} 2)+(1 / \mathrm{b}(\mathrm{t})-1 / \mathrm{b}(\mathrm{t}) \wedge 3) * \operatorname{rp}(\mathrm{t}) / \operatorname{sqrt}\left(\mathrm{I}_{-} \mathrm{r} 2(\mathrm{t})\right)\right)$;

$$
\frac{\phi(u) \phi\left(\frac{\mathrm{sq}(t) u}{\operatorname{nsigma}(t)}\right)\left(\left(\frac{\operatorname{nsigma}(t)}{\mathrm{sq}(t) u}-\frac{\operatorname{nsigma}(t)^{3}}{\mathrm{sq}(t)^{3} u^{3}}\right) \sqrt{\lambda 2}+\frac{\left(\frac{1}{\mathrm{~b}(t)}-\frac{1}{\mathrm{~b}(t)^{3}}\right) \operatorname{rp}(t)}{\sqrt{\mathrm{I} \_2(t)}}\right)}{\sqrt{2 \pi}}
$$

$>$ SA1:=simplify(simplify(series $(A 1(t), t=0,6)$, siderels), power);

$$
S A 1:=\frac{1}{16} \frac{\sqrt{2} e^{\left(-1 / 2 \frac{u^{2}\left(\mu 4+\lambda 2^{2}\right)}{\mu 4}\right)} \mu 4^{(5 / 2)}}{\lambda 2^{(7 / 2)} \pi^{(3 / 2)} u^{3}} t^{2}+\mathrm{O}\left(t^{4}\right)
$$

with assumptions on $t, \mu 4$ and $\lambda 2$

Expansion of the exponent for using Lemma 4.3 (ii), $\mathrm{p}=2$

$>\quad \mathrm{L} 2:=\mathrm{t}->(1-\mathrm{r}(\mathrm{t})) /\left((1+\mathrm{r}(\mathrm{t})) * \mathrm{nsigma}(\mathrm{t})^{\wedge} 2\right)-($ lambda4 $4-\mathrm{mu} 4) / \mathrm{mu} 4$;

$$
L 2:=t \rightarrow \frac{1-\mathrm{r}(t)}{(1+\mathrm{r}(t)) \mathrm{nsigma}(t)^{2}}-\frac{\lambda 4-\mu 4}{\mu 4}
$$

$>$ SL2: $=$ simplify ( $\operatorname{simplify}(\operatorname{series}(\mathrm{L} 2(\mathrm{t}), \mathrm{t}=0,6)$, siderels $)$, power $)$;

$$
S L 2:=\frac{1}{18} \frac{\lambda 2 \mu 6}{\mu 4^{2}} t^{2}+\mathrm{O}\left(t^{4}\right)
$$

with assumptions on $t, \lambda 2, \mu 6$ and $\mu 4$

We define $c$ as the square root of the coefficient of $t^{\wedge} 2$

$c:=\operatorname{sqrt}($ op $(1$, SL2 $))$

$$
c:=\frac{1}{6} \frac{\sqrt{2} \sqrt{\lambda 2 \mu 6}}{\mu 4}
$$

with assumptions on $\lambda 2, \mu 6$ and $\mu 4$

$>\operatorname{nu} 1 \mathrm{~b}:=(\operatorname{sqrt}(2 * \mathrm{pi})) * \mathrm{op}(1, \mathrm{SA} 1) *\left(\mathrm{c}^{\wedge}(-3) * \mathrm{u}^{\wedge}(-3) / 2\right)$;

$$
n u 1 b:=\frac{27}{8} \frac{\sqrt{2} e^{\left(-1 / 2 \frac{u^{2}\left(\mu 4+\lambda 2^{2}\right)}{\mu 4}\right)} \mu 4^{(11 / 2)}}{\pi \lambda 2^{(7 / 2)} u^{6}(\lambda 2 \mu 6)^{(3 / 2)}}
$$

with assumptions on $\mu 4, \lambda 2$ and $\mu 6$

PROOF OF THE EQUIVALENT OF NU2

$>\quad \mathrm{m} 1:=\mathrm{t}->(1+\mathrm{rho}(\mathrm{t})) * 2 * \operatorname{sigma}(\mathrm{t})^{\wedge} 2 /\left(\mathrm{pi} * \mathrm{~b}(\mathrm{t}) * \operatorname{sqrt}\left(I_{-} \mathrm{r} 2(\mathrm{t})\right)\right)$;

$$
m 1:=t \rightarrow 2 \frac{(1+\rho(t)) \sigma(t)^{2}}{\pi \mathrm{b}(t) \sqrt{\mathrm{I} \_\mathrm{r} 2(t)}}
$$

$>\operatorname{sm1}:=\operatorname{simplify}(\operatorname{simplify}(\operatorname{series}(m 1(t), t=0,8)$, siderels $)$, power $)$;

$$
s m 1:=\frac{1}{36} \frac{\mu 6 \sqrt{\mu 4}}{\lambda 2^{(5 / 2)} \pi u} t^{3}+\mathrm{O}\left(t^{5}\right)
$$

with assumptions on $t, \mu 6, \mu 4$ and $\lambda 2$ 
$>\mathrm{m} 2:=\mathrm{t}->(-4 / \mathrm{pi}) * \operatorname{sigma}(\mathrm{t})^{\wedge} 2 * \mathrm{~b}(\mathrm{t})^{\wedge}(-3) / \operatorname{sqrt}\left(I_{-} \mathrm{r} 2(\mathrm{t})\right)$;

$$
m 2:=t \rightarrow-4 \frac{\sigma(t)^{2}}{\pi \mathrm{b}(t)^{3} \sqrt{\mathrm{I} \_r 2(t)}}
$$

$>$ sm2:=simplify $(\operatorname{simplify}(\operatorname{series}(m 2(t), t=0,6)$, siderels $)$, power $)$;

$$
s m 2:=-\frac{\mu 4^{(5 / 2)}}{\pi u^{3} \lambda 2^{(7 / 2)}} t+\mathrm{O}\left(t^{3}\right)
$$

with assumptions on $t, \mu 4$ and $\lambda 2$

$>\quad \mathrm{m} 3:=\mathrm{t}->-(1+\mathrm{rho}(\mathrm{t})) * \operatorname{sigma}(\mathrm{t})^{\wedge} 2 * \mathrm{k}(\mathrm{t}) /\left(\operatorname{pi} * \operatorname{sqrt}\left((2 * \mathrm{pi}) * \mathrm{I} \_r 2(\mathrm{t})\right)\right)$;

$$
m 3:=t \rightarrow-\frac{(1+\rho(t)) \sigma(t)^{2} \mathrm{k}(t)}{\pi \sqrt{2 \pi \mathrm{I} \_r 2(t)}}
$$

$>$ sm3:=simplify $(\operatorname{simplify}(\operatorname{series}(m 3(t), t=0,6)$, siderels $)$, power $)$;

$$
s m 3:=-\frac{1}{864} \frac{\mu 6^{(3 / 2)} \sqrt{2}}{\lambda 2^{2} \sqrt{\mu 4} \pi^{(3 / 2)}} t^{4}+\mathrm{O}\left(t^{6}\right)
$$

with assumptions on $t, \mu 6, \lambda 2$ and $\mu 4$

$>\quad \mathrm{m} 4:=\mathrm{t}->(2 / \mathrm{pi}) * \operatorname{sigma}(\mathrm{t}) \wedge 2 * \mathrm{k}(\mathrm{t}) \wedge 3 / \operatorname{sqrt}\left(2 * \mathrm{pi} * \mathrm{I} \_r 2(\mathrm{t})\right)$;

$$
m_{4}:=t \rightarrow 2 \frac{\sigma(t)^{2} \mathrm{k}(t)^{3}}{\pi \sqrt{2 \pi \mathrm{I} \_r 2(t)}}
$$

$>$ sm4:=simplify ( $\operatorname{simplify}(\operatorname{series}(m 4(t), t=0,6)$, siderels $)$, power $)$;

$$
s m_{4}:=\frac{1}{864} \frac{\mu 6^{(3 / 2)} \sqrt{2}}{\lambda 2^{2} \sqrt{\mu 4} \pi^{(3 / 2)}} t^{4}+\mathrm{O}\left(t^{6}\right)
$$

with assumptions on $t, \mu 6, \lambda 2$ and $\mu 4$

$>\quad \mathrm{m} 5:=\mathrm{t}->(4 / \mathrm{pi}) * \operatorname{sigma}(\mathrm{t}) \wedge 2 * \mathrm{k}(\mathrm{t}) * \mathrm{~b}(\mathrm{t})^{\wedge}(-2) / \operatorname{sqrt}\left(2 * \mathrm{pi} * \mathrm{I} \_r 2(\mathrm{t})\right)$;

$$
m 5:=t \rightarrow 4 \frac{\sigma(t)^{2} \mathrm{k}(t)}{\pi \mathrm{b}(t)^{2} \sqrt{2 \pi \mathrm{I} \_r 2(t)}}
$$

$>$ sm5:=simplify (simplify $(\operatorname{series}(m 5(t), t=0,6)$, siderels $)$, power $)$;

$$
s m 5:=\frac{1}{12} \frac{\sqrt{\mu 6} \mu 4^{(3 / 2)} \sqrt{2}}{\lambda 2^{3} \pi^{(3 / 2)} u^{2}} t^{2}+\mathrm{O}\left(t^{4}\right)
$$

with assumptions on $t, \mu 6, \mu 4$ and $\lambda 2$

$>112:=t->(b(t) / u)^{-2}+2 /(1+r(t))-l a m b d a 4 / m u 4 ;$

$$
l 12:=t \rightarrow \frac{\mathrm{b}(t)^{2}}{u^{2}}+2 \frac{1}{1+\mathrm{r}(t)}-\frac{\lambda 4}{\mu 4}
$$

$>$ simplify(simplify(series $(112(t), t=0,8)$, siderels), power);

$$
\frac{1}{18} \frac{\lambda 2 \mu 6}{\mu 4^{2}} t^{2}+\mathrm{O}\left(t^{4}\right)
$$

with assumptions on $t, \lambda 2, \mu 6$ and $\mu 4$

$>122:=t->\left((b(t) / u)^{\wedge} 2\right) *\left(1+k(t)^{\wedge} 2\right)+2 /(1+r(t))-l a m b d a 4 / m u 4 ;$

$$
\text { l22 }:=t \rightarrow \frac{\mathrm{b}(t)^{2}\left(1+\mathrm{k}(t)^{2}\right)}{u^{2}}+2 \frac{1}{1+\mathrm{r}(t)}-\frac{\lambda 4}{\mu 4}
$$


$>$ simplify(simplify(series $(122(t), t=0,8)$, siderels), power);

$$
\begin{aligned}
& \frac{1}{12} \frac{\lambda 2 \mu 6}{\mu 4^{2}} t^{2}+\mathrm{O}\left(t^{4}\right) \\
& \quad \text { with assumptions on } t, \lambda 2, \mu 6 \text { and } \mu 4
\end{aligned}
$$

$>\operatorname{simplify}\left(\operatorname{int}\left(\cos (t)^{\wedge} 3, t=0 \ldots \arctan (\operatorname{sqrt}(2) / 2)\right)\right.$, power $)$;

$$
\frac{8}{27} \sqrt{3}
$$

$>\quad o p m 1:=o p(1, \mathrm{sm} 1)$

$$
\text { opm1 }:=\frac{1}{36} \frac{\mu 6 \sqrt{\mu 4}}{\lambda 2^{(5 / 2)} \pi u}
$$

with assumptions on $\mu 6, \mu 4$ and $\lambda 2$

$>\quad o p m 2:=o p(1, \mathrm{sm} 2)$;

$$
\text { opm2 }:=-\frac{\mu 4^{(5 / 2)}}{\pi u^{3} \lambda 2^{(7 / 2)}}
$$

with assumptions on $\mu 4$ and $\lambda 2$

$>\quad o p m 5:=o p(1, \mathrm{sm} 5)$;

$$
\text { opm } 5:=\frac{1}{12} \frac{\sqrt{\mu 6} \mu 4^{(3 / 2)} \sqrt{2}}{\lambda 2^{3} \pi^{(3 / 2)} u^{2}}
$$

with assumptions on $\mu 6, \mu 4$ and $\lambda 2$

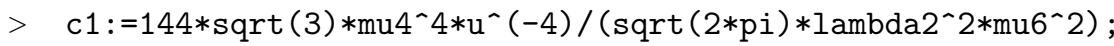

$$
c 1:=72 \frac{\sqrt{3} \mu 4^{4} \sqrt{2}}{u^{4} \sqrt{\pi} \lambda 2^{2} \mu 6^{2}}
$$

with assumptions on $\mu 4, \lambda 2$ and $\mu 6$

$>\quad c 2:=3 * \operatorname{sqrt}(3) * m u 4^{\wedge} 2 * u^{\wedge}(-2) /(\operatorname{sqrt}(2 * p i) * \operatorname{lambda} 2 * m u 6)$;

$$
c 2:=\frac{3}{2} \frac{\sqrt{3} \mu 4^{2} \sqrt{2}}{u^{2} \sqrt{\pi} \lambda 2 \mu 6}
$$

with assumptions on $\mu 4, \lambda 2$ and $\mu 6$

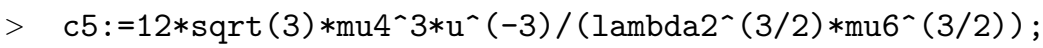

$$
c 5:=12 \frac{\sqrt{3} \mu 4^{3}}{u^{3} \lambda 2^{(3 / 2)} \mu 6^{(3 / 2)}}
$$

with assumptions on $\mu 4, \lambda 2$ and $\mu 6$

$>\quad \mathrm{B}:=\mathrm{opm} 1 * \mathrm{c} 1+\mathrm{opm} 2 * \mathrm{c} 2+\mathrm{opm} 5 * \mathrm{c} 5$;

$$
B:=\frac{3}{2} \frac{\mu 4^{(9 / 2)} \sqrt{3} \sqrt{2}}{\pi^{(3 / 2)} u^{5} \lambda 2^{(9 / 2)} \mu 6}
$$

with assumptions on $\mu 4, \lambda 2$ and $\mu 6$

$>\operatorname{simplify}(\mathrm{B})$;

$$
\begin{aligned}
& \frac{3}{2} \frac{\mu 4^{(9 / 2)} \sqrt{3} \sqrt{2}}{\pi^{(3 / 2)} u^{5} \lambda 2^{(9 / 2)} \mu 6} \\
& \quad \text { with assumptions on } \mu 4, \lambda 2 \text { and } \mu 6
\end{aligned}
$$

